\title{
Research on Consumer 's Purchase Behavior of Green Product
}

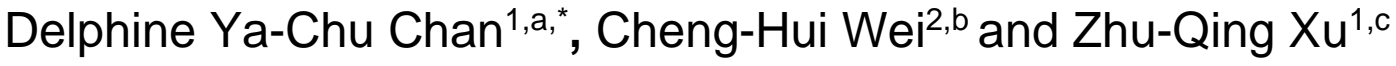 \\ ${ }^{1}$ School of Business, Beijing Institute of Technology, Zhuhai, Guangdong, China \\ ${ }^{2}$ Nanfang College of Sun Yat-Sen University, Guangzhou, Guangdong, China \\ aw-chu119@qq.com, bw-hui223@qq.com, c2970752822@qq.com \\ ${ }^{*}$ Corresponding author
}

Keywords: Environmental consciousness, Purchase behavior, Social responsibility, Attitude.

\begin{abstract}
In view of the economic potential of green consumption, this study collected 555 valid samples through questionnaires to explore the influencing factors of consumers' green purchase behavior. The results show that consumer's perception of corporate social responsibility (CSR) and attitude toward green product have significantly positive effects on the green purchase behavior. Among them, the green attitude has partially mediating effect on the relationship between perceived CSR and green purchase behavior. In addition, environmental consciousness has a significantly positive moderating effect on the relationship between green attitudes and green purchase behavior, and it has a significantly negative moderating effect on the relationship between perceived CSR and attitudes toward green product. It can be seen that perceived CSR, green attitude, and environmental consciousness are the important factors influencing consumers' green purchase behavior.
\end{abstract}

\author{
消费者绿色购买行为研究 \\ 詹雅竹 ${ }^{1, a,{ }^{*}}$, 魏征辉 $2, \mathrm{~b}$, 许竹青 ${ }^{1, \mathrm{c}}$ \\ 1 北京理工大学珠海学院商学院, 珠海, 广东, 中国 \\ 2中山大学南方学院, 广州, 广东, 中国 \\ aw-chu119@qq.com, bw-hui223@qq.com, c2970752822@qq.com \\ *通讯作者
}

关键词: 环境意识; 购买行为; 社会责任; 态度

中文摘要. 鉴于绿色消费的经济潜力, 本研究通过问卷调查法收集了 555 份有效问卷，探讨消 费者绿色购买行为的影响因素。结果表明, 消费者的感知企业社会责任和绿色产品态度都能 正向促进其绿色购买行为; 其中，绿色态度在感知企业社会责任和绿色购买行为的关系中更 具有部分中介效果。此外, 环境意识在绿色态度对绿色购买行为的关系中具有显著的正向调 节作用, 而在感知企业社会责任对绿色态度的关系中则具有显著的负向调节作用, 由此可知, 感知企业社会责任、绿色态度、环境意识是消费者绿色购买行为的重要影响因素。

1. 引言

全球环境问题逐年严峻，绿色消费成为节能减碳的关键环节，也是各国消费趋势之一 ${ }^{[1] 。}$ 因此, 企业开始从产品研发、生产至销售等供应链过程减少对环境的破坏, 并作为履行社会 责任与关怀消费者的卖点。在过去研究中, 学者们从消费者的角度出发, 探讨其对绿色产品 
消费的影响因素，包括消费者自身的客观因素、行为心理、生活方式等内部因素，以及社会 文化等外部因素 ${ }^{[2-5]}$, 也发现社会责任感较高的消费者对绿色消费的行动会高于社会责任感较 低者 ${ }^{[6]}$, 但是探讨社会责任对绿色消费的影响研究仍不多。综上所述, 本文希望探究消费者 的感知社会责任、绿色态度与环境意识对绿色购买行为的影响，为企业提供管理意涵。

\section{2. 文献探讨}

\section{1 感知企业社会责任、绿色态度与绿色购买行为}

感知企业社会责任(perceived corporate social responsibility, perceived CSR)是消费者对于 企业执行社会责任的态度和行为表现的综合印象和整体评价，能反映消费者对企业责任态度 和行为的敏感度和认知度 ${ }^{[7-8]}$ 。绿色态度(attitudes toward green product)则是消费者对绿色产品 所持有的心理倾向 ${ }^{[9-10]}$ 。绿色购买行为(green purchase behavior)作为一种环境责任行为 ${ }^{[11]}$, 是 指消费者在购买过程中以环境意识为导向对产品的环保属性及特点进行考虑及购买的消费行 为 ${ }^{[12-14]}$ 。过去研究显示, 消费者对绿色产品的态度会受到CSR信息的影响, 尤其是正面的CSR 信息会使其认为企业的产品质量高, 并提高对该企业或产品的评价, 进而影响对该产品的购 买行为 ${ }^{[6,15-16]}$ 。故本研究提出以下假设:

H1：感知CSR对(a)绿色态度、(b)绿色购买行为有显著影响。

$\mathrm{H} 2$ : 绿色态度在感知CSR 和绿色购买行为之间具有中介作用。

\section{2 环境意识}

环境意识(environmental consciousness)是基于认知层面的角度定义为个人对环境保护的 关注程度 ${ }^{[17]}$ 。过去研究显示, 消费者感知的CSR对绿色态度和绿色购买行为有显著影响 ${ }^{[6]}$, 环境意识对绿色态度和绿色购买行为也存在影响作用 ${ }^{[18-20]}$; 其中, 环境意识愈高的消费者, 对绿色产品的了解程度与积极性也愈高, 但也可能因为既定的刻板印象而影响消费行为 ${ }^{[21]}$ 。 故本研究提出以下假设:

H3: 环境意识在(a)感知CSR 和绿色购买行为的关系中、(b)感知CSR和绿色态度的关系中、 (c)绿色态度和绿色购买行为的关系中具有调节作用。

\section{3. 研究方法}

本研究采用问卷调查法，调查对象是广东地区的消费者。变量测题选自先前研究，如表1

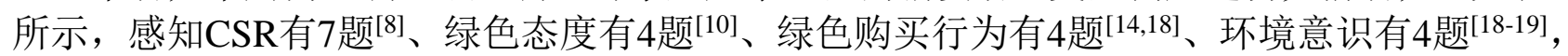
均使用Likert 5 点量表。本次调查共回收 560 份问卷, 剔除5份无效样本，有效样本数555份, 回收率达到 $99.1 \%$, 在置信区间 $95 \%$ 、误差水平 $5 \%$ 的情况下，达到抽样容量要求。其中男性 占 $42.16 \%$, 女性占 $57.84 \%$; 受测者多为 $21-30$ 岁(62.88\%), 收入以 3000 元(含)以下最多 $(39.64 \%)$, 教育程度则以大学为主(73.69\%)。

\section{4. 分析结果}

\section{1 信效度分析}

从表 1 可知, 所有变量的Cronbach's $\alpha>0.7$, 项目总相关 $>0.5$, 因子载荷 $>0.7$, 组成信度 $>0.7$, AVE $>0.5$, 显示变量测题具有高度的内部一致性和良好的收玫效度。表 2 可见AVE开方后均大 于变量间的相关系数, 显示变量间也有良好的区别效度。 
表1 信度与收敛效度

\begin{tabular}{|c|c|c|c|c|c|}
\hline \multirow{2}{*}{ Construct \& Item } & \multicolumn{2}{|c|}{ Internal reliability } & \multicolumn{3}{|c|}{ Convergent validity } \\
\hline & Cronbach's $\alpha$ & Item-total correlation & Factor loading & $\mathrm{CR}$ & AVE \\
\hline \multicolumn{6}{|c|}{ Perceived CSR $(M=3.932 ; \mathbf{S D}=.622)$ from Lombart \& Louis $(2014)^{[8]}$} \\
\hline CSR1. 公司整体尽量达到环保 & .886 & .631 & .731 & .911 & .594 \\
\hline CSR2. 公平对待消费者 & & .672 & .767 & & \\
\hline CSR3. 提供准确的产品信息 & & .682 & .776 & & \\
\hline CSR4. 重视消费者权益 & & .728 & .813 & & \\
\hline CSR5. 支持地方政府环保 & & .654 & .751 & & \\
\hline CSR6. 支持社会文化活动 & & .671 & .766 & & \\
\hline CSR7. 支持社会慈善事业 & & .695 & .786 & & \\
\hline \multicolumn{6}{|c|}{ Green Attitudes (M=4.170; SD=.592) from Chang $(2011)^{[10]}$} \\
\hline ATT1. 喜欢绿色产品 & .797 & .614 & .797 & .868 & .623 \\
\hline ATT2. 对绿色产品持正面态度 & & .661 & .830 & & \\
\hline ATT3. 觉得绿色产品对环境好 & & .610 & .794 & & \\
\hline ATT4. 购买绿色产品感到骄傲 & & .540 & .732 & & \\
\hline \multicolumn{6}{|c|}{ Purchase Behavior $(\mathbf{M}=3.925 ; \mathbf{S D}=. \mathbf{6 1 0})$ from Kim \& Choi $(2005)^{[14]}$ and Matthes et al. $(2014)^{[18]}$} \\
\hline PB1. 购买产品有生物降解包装 & .772 & .564 & .762 & .854 & .595 \\
\hline $\mathrm{PB} 2$. 愿意购买绿色产品 & & .557 & .757 & & \\
\hline PB3. 我可能转向购买绿色产品 & & .626 & .810 & & \\
\hline PB4. 选对环境伤害较少的产品 & & .547 & .754 & & \\
\hline \multicolumn{6}{|c|}{ Environmental Consciousness (M=4.177; SD=.582) from Matthes et al. $(2014)^{[18]}$ and Ritter et al. $(2014)^{[19]}$} \\
\hline EC1. 关心生态环境 & .817 & .683 & .837 & .880 & .647 \\
\hline EC2. 关心地球的未来发展 & & .678 & .833 & & \\
\hline EC3. 觉得自己是环境中的一员 & & .632 & .800 & & \\
\hline EC4. 认为环境会影响生活质量 & & .563 & .744 & & \\
\hline
\end{tabular}

EC4. 认为环境会影响生活质量

表2 区别效度

\begin{tabular}{lcccc}
\hline \multicolumn{1}{c}{ Constructs } & 1 & 2 & 3 & 4 \\
\hline 1. CSR & $\mathbf{. 7 7 1}$ & & & \\
2. ATT & $.574^{* *}$ &. $\mathbf{7 8 9}$ & & \\
3. PB & $.622^{* *}$ & $.683^{* *}$ &. $\mathbf{7 7 1}$ & $\mathbf{. 0 4}$ \\
4. EC & $.533^{* *}$ & $.644^{* *}$ & $.603^{* *}$ & \\
\hline
\end{tabular}

Note: The square roots of average variance extracted (AVEs) are shown in bold on the diagonal of the matrix; off diagonal elements are the correlations between constructs.

\section{2 回归分析}

回归分析结果显示, 感知 CSR 对绿色态度 $(\beta=.574, p<.001)$ 和绿色购买行为 $(\beta=.622, p<.001)$ 皆有显著的正向影响, 且调整后的解释力分别达到 $32.8 \%$ 和 $38.6 \%$, 两者的 $F=271.274$ 和 $F=348.693$ 也达到显著水平 $(p<.001)$, 因此H1(a)、H1(b)获得支持。在感知CSR与绿色购买行为 的关系中加入绿色态度时, 感知CSR对绿色购买行为的影响下降但仍显著 $(\beta=.342, p<.001)$, 而且调整后的解释力达到 $54.4 \%, F=331.727$ 也达到显著水平 $(p<.001)$, 可知绿色态度在感知 $\mathrm{CSR}$ 与绿色购买行为之间具有部分中介效果，故 $\mathrm{H} 2$ 获得支持。

在调节效果方面, 首先, 由于感知CSR $(\beta=.420, p<.001)$ 与环境意识 $(\beta=.380, p<.001)$ 对于 绿色购买行为皆呈现正向显着关系, 但感知 CSR与环境意识的相乘项对绿色购买行为的影响 未达到显着水平 $(\beta=.006, p>.05)$, 而且调整后的解释力达到 $48.7 \%, F=176.590$ 达到显著水平 ( $p<.001)$, VIF值介于1.111 1.396, 没有共线性问题, 表示感知CSR对绿色购买行为的正向关 系, 不会受到消费者的环境意识影响而使上述关系产生显着差异, 即H3(a)未获得支持。其次, 感知 CSR $(\beta=.322, p<.001)$ 与环境意识 $(\beta=.472, p<.001)$ 对于绿色态度皆呈现正向显着关系, 而 感知CSR与环境意识的相乘项对绿色态度之关系也达到显着的负向影响 $(\beta=-.073, p<.05)$, 调整 后的解释力为 $49.1 \%, F=179.098$ 也达到显著水平 $(p<.001)$, VIF值介于 $1.111 \sim 1.498$, 没有共线 性问题, 表示感知CSR对绿色态度的正向关系, 会因为消费者本身的环境意识而使上述关系 产生弱化效果, 即 $\mathrm{H} 3(\mathrm{~b})$ 获得支持。最后, 绿色态度 $(\beta=.504, p<.001)$ 与环境意识 $(\beta=.279, p<.001)$ 对绿色购买行为皆呈现显着的正向关系, 而绿色态度与环境意识的相乘项对绿色购买行为之 关系也达到正向显着影响 $(\beta=.086, p<.01)$, 而且调整后的解释力为 $51.7 \%, F=198.326$ 达到显著 
水平 $(p<.001), \mathrm{VIF}$ 值介于 $1.147 \sim 1.754$, 没有共线性问题, 表示绿色态度对于绿色购买行为的 正向关系，会因为消费者环境意识而使上述关系产生强化效果，即 $\mathrm{H} 3(\mathrm{c})$ 获得支持。

\begin{tabular}{|c|c|c|c|c|c|c|c|}
\hline Hypothesis & Path & $\beta$ & $p$ & $R^{2}$ & $F$ & Sig. & Supported \\
\hline H1(a) & $\mathrm{CSR} \rightarrow \mathrm{ATT}$ & .574 & $<.001$ & $32.8 \%$ & 271.274 & $<.001$ & $\mathrm{Y}$ \\
\hline H1(b) & $\mathrm{CSR} \rightarrow \mathrm{PB}$ & .622 & $<.001$ & $38.6 \%$ & 348.693 & $<.001$ & $\mathrm{Y}$ \\
\hline $\mathrm{H} 2$ & $\mathrm{CSR} \rightarrow \mathrm{ATT} \rightarrow \mathrm{PB}$ & .342 & $<.001$ & $54.4 \%$ & 331.727 & $<.001$ & $\mathrm{Y}$ \\
\hline \multirow{3}{*}{$\mathrm{H} 3(\mathrm{a})$} & $\mathrm{CSR} \rightarrow \mathrm{PB}$ & .420 & $<.001$ & \multirow{3}{*}{$48.7 \%$} & \multirow{3}{*}{176.590} & \multirow{3}{*}{$<.001$} & \multirow{3}{*}{$\mathrm{N}$} \\
\hline & $\mathrm{EC} \rightarrow \mathrm{PB}$ & .380 & $<.001$ & & & & \\
\hline & $\mathrm{CSR} * \mathrm{EC} \rightarrow \mathrm{PB}$ & .006 & $>.05$ & & & & \\
\hline \multirow{3}{*}{$\mathrm{H} 3(\mathrm{~b})$} & $\mathrm{CSR} \rightarrow \mathrm{ATT}$ & .322 & $<.001$ & \multirow{3}{*}{$49.1 \%$} & \multirow{3}{*}{179.098} & \multirow{3}{*}{$<.001$} & \multirow{3}{*}{$\mathrm{Y}$} \\
\hline & $\mathrm{EC} \rightarrow \mathrm{ATT}$ & .472 & $<.001$ & & & & \\
\hline & $\mathrm{CSR} * \mathrm{EC} \rightarrow \mathrm{ATT}$ & -.073 & $<.05$ & & & & \\
\hline \multirow{3}{*}{$\mathrm{H} 3(\mathrm{c})$} & $\mathrm{ATT} \rightarrow \mathrm{PB}$ & .504 & $<.001$ & \multirow{3}{*}{$51.7 \%$} & \multirow{3}{*}{198.326} & \multirow{3}{*}{$<.001$} & \multirow{3}{*}{$\mathrm{Y}$} \\
\hline & $\mathrm{EC} \rightarrow \mathrm{PB}$ & .279 & $<.001$ & & & & \\
\hline & $\mathrm{ATT}^{*} \mathrm{EC} \rightarrow \mathrm{PB}$ & .086 & $<.01$ & & & & \\
\hline
\end{tabular}

\section{5. 结论与管理意涵}

消费者的感知CSR对绿色购买行为有显著影响，故企业应从以下三方面提升消费者对 CSR的感知：（1)使企业整体尽可能达到社会责任, 带给消费者一个好的企业形象; (2)注重对 消费者的责任，公平对待所有消费者; (3)支持地方政府环保、社会文化活动、社会慈善事业。 此外, 消费者的环境意识也会调节消费者的绿色态度进而促进其绿色购买行为, 因此企业在 营销宣传时, 应适度将产品的绿色信息有效地传递给消费者, 近而培养消费者的绿色态度与 环境意识，促进企业的可持续发展。

\section{致谢}

本文为广东省高校创新人才类项目（人文社科） 《基于可持续发展的组织营销与消费研 究》(2015WQNCX191)的阶段性成果之一。

\section{References}

[1] M. C. Olsen, R. J. Slotegraaf, and S. R. Chandukala, Green claims and message frames: how green new products change brand attitude, J. Marketing, vol. 78, pp. 119-137, 2014.

[2] G. Levin, Consumers turning green: jwt survey, Advertising Age, vol. 61, pp.74, 1990.

[3] F. Guo, Analysis on the mechanism of the influence factors of green consumption behavior, Beijing Forestry University, 2013.

[4] Z. Y. He and S. Q. Yang, A Study about life style characteristics of green consumer, Nankai Business Review, vol. 7, pp. 4-10, 2004.

[5] H. J. Lee and S. Y. Park, Environmental orientation in going green: A qualitative approach to consumer psychology and sociocultural factors of green consumption, J. Global Scholars of Marketing Sci., vol. 23, pp. 245-262, 2013.

[6] G. Berens, C. B. M. V. Riel, and G. H. V. Bruggen, Corporate associations and consumer product responses: the moderating role of corporate brand dominance, J. Marketing, vol. 69, pp. 35-48, 2013.

[7] Z. Q. Li and Y. M. Lu, The influences of consumers' corporate social responsibility perception on brand equity, Economy \& Management, vol.14, pp. 84-86, 2015. 
[8] C. Lombart and D. Louis, A study of the impact of corporate social responsibility and price image on retailer personality and consumers' reactions (satisfaction, trust and loyalty to the retailer), J. Retail. \& Cons. Serv., vol.21, pp. 630-642, 2014.

[9] A. H. Eagly and S. Chaiken, The Psychology of Attitudes, Orlando, FL: Harcourt Brace Jovanovich, 1993.

[10] C. Chang, Feeling ambivalent about going green: implications for green advertising processing, J. Advertising, vol.40, pp.19-31, 2011.

[11] I. Balderjahn, Personality variables and environmental attitudes as predictors of ecologically responsible consumption patterns, J. Bus. Res., vol.17, pp. 51-56, 1988.

[12] T. Mainieri, E. G. Barnett, T. R. Valdero, J. B. Unipan, and S. Oskamp, Green buying: the influence of environmental concern on consumer behavior, J. Soc. Psychol, vol.137, pp. 189-204, 1997.

[13] L. J. Shrum, J. A. McCarty and T. M. Lowrey, Buyer characteristics of the green consumer and their implications for advertising strategy, J. Advertising, vol.24, pp. 71-82, 1995.

[14] Y. Kim, S.M. Choi, Antecedents of green purchase behavior: an examination of collectivism, environmental concern, and PCE, Adv. Cons. Res., vol.32, pp.592-599, 2005.

[15] S. Sen, C. B. Bhattacharya and D. Korschun, The role of corporate social responsibility in strengthening multiple stakeholder relationships: a field experiment, J. Acad. Market. Sci., vol.34, pp. 158-166, 2006.

[16] C. P. Lin, S. C. Chen, C. K. Chiu and W. Y. Lee, Understanding purchase intention during product-harm crises: moderating effects of perceived corporate ability and corporate social responsibility, J. Bus. Ethics, vol.102, pp.455-471, 2011.

[17] R. E. Dunlap and R. E. Jones, Environmental concern: Conceptual and measurement issues, Westport, CT: Greenwood Press, 2002.

[18] J. Matthes, A. Wonneberger and D. Schmuck, Consumers' green involvement and the persuasive effects of emotional versus functional ads, J. Bus. Res., vol.67, pp. 1885-1893, 2014.

[19] M. Ritter, M. Borchardt, G. L. R. Vaccaro, G. M. Pereira, and F. Almeida, Motivations for promoting the consumption of green products in an emerging country: exploring attitudes of brazilian consumers, J. Cleaner Production, vol.106, pp. 507-520, 2014.

[20] X. L. Chen, D. Y. C. Chan, and C. H. Wei, The research on environmental conscious and green consumption behavior in China, Serv. Sci. and Manage., vol. 4, pp. 30-36, 2015.

[21] Y. C. Lin and C. A. Chang, Double standard: the role of environmental consciousness in green product usage, J. Marketing, vol. 76, pp. 125-134, 2012. 